IOS Press

\title{
Association between physical activity and pain processing in adults with chronic low back pain compared to pain-free controls
}

\author{
Lindsay C. Orr ${ }^{\mathrm{a}, *}$, Steven Z. George ${ }^{\mathrm{b}}$ and Corey B. Simon ${ }^{\mathrm{a}, \mathrm{c}}$ \\ ${ }^{a}$ Department of Physical Therapy, College of Public Health and Health Professions, University of Florida, \\ Gainesville, FL, USA \\ ${ }^{\mathrm{b}}$ Duke Clinical Research Institute, Department of Orthopaedic Surgery, Duke University, Durham, NC, USA \\ ${ }^{c}$ Pain Research \& Intervention Center of Excellence, University of Florida, Gainesville, FL, USA
}

\begin{abstract}
.
BACKGROUND: Pain sensitivity has been negatively associated with physical activity levels. Few studies have examined associations between experimentally induced pain sensitivity and physical activity in adults with chronic low back pain and painfree controls.

OBJECTIVE: The objective of this study was to examine associations between physical activity levels and how an individual processes pain using experimentally induced pain stimuli.

METHODS: Seventy subjects $(\mathrm{CLBP}=49$; mean age $=46.8 \pm 14.9$; Pain-free $=21$; mean age $=45.3 \pm 18.2$, $\mathrm{n}$ of females $=$ 46) participated. A self-report questionnaire derived from the International Physical Activity Questionnaire (IPAQ) was used to calculate an activity level index. Pain sensitivity was assessed via quantitative sensory testing (QST) at the right lower extremity. RESULTS: Moderate $(\mathrm{U}=688, p<0.05)$ and vigorous $(\mathrm{U}=649, p<0.05)$ physical activity levels were higher in pain-free vs. individuals with CLBP. Activity level was not associated with pain sensitivity (Pain-free: R2 $=0.02, p>0.05$; CLBP: R2 $=0.01, p>0.05)$. Both moderate $(\mathrm{R} 2=0.49, p<0.05)$ and vigorous $(\mathrm{R} 2=0.68, p<0.01)$ physical activity were associated with pain modulation amongst pain-free individuals.

CONCLUSIONS: Findings suggest that physical activity influences pain modulation amongst pain-free individuals, however no relationship exists once CLBP is present. However, future investigation will elucidate the extent to which physical activity level either prevents CLBP or is effective in alleviating CLBP.
\end{abstract}

Keywords: Physical activity, pain modulation, chronic low back pain

\section{Background}

Chronic low back pain (CLBP) is one of the most prevalent pain conditions and the leading cause of chronic disability worldwide [1,2]. Elucidating mechanisms of CLBP chronicity is a research priority since

\footnotetext{
${ }^{*}$ Corresponding author: Lindsay C. Orr, Master of Occupational Therapy, University of Florida Department of Physical Therapy, PO Box 100154, 101 S. Newell Drive, Gainesville, FL 32610, USA. Tel.: +1 352273 6085; Fax: +1 352273 6109; E-mail: lindsaycorr@ ufl.edu.
}

such factors can be instrumental in clinical rehabilitation. Recent work has determined numerous factors that influence the LBP experience, including age, sex, smoking, and psychological distress [3-6]. Physical activity (e.g. exercise, recreation) is also believed to play a role; however, the extent to which physical activity prevents, alleviates, and/or exacerbates LBP is undetermined $[7,8]$.

Limitations in the previous physical activity research have prevented researchers from determining the direction and magnitude of influence between physical activity and CLBP. For example, individuals

ISSN 1053-8127/17/\$35.00 (C) 2017 - IOS Press and the authors. All rights reserved

This article is published online with Open Access and distributed under the terms of the Creative Commons Attribution Non-Commercial License (CC-BY-NC 4.0). 
with mild CLBP may have an increased probability of maintaining recreational activities, while those with severe CLBP may compensate or avoid activities [8]. In such cases, it would be difficult to determine whether physical activity improved CLBP or was dependent on the person's pain state. Another issue is that the association between physical activity and LBP may not be linear. Vigorous activity may be just as detrimental to pain chronicity as maintaining a sedentary lifestyle, whereas a moderate physical activity level would be more beneficial [8,9]. Limitations in prior research impede translation to clinical care. Although physical activity is often championed by health care professionals to reduce health risks [10], physical activity among individuals with CLBP may be attenuated - or worse, discouraged - if the assumption is that physical activity has deleterious effects.

Pain researchers have commonly used laboratorycontrolled experimental pain evocation to assess pain processing changes in the presence of chronic pain conditions [11-13]. A large body of research exists to show that individuals with CLBP have lower pain thresholds to pressure or punctate stimuli compared to pain-free controls [14-17] which suggests that CLBP is associated with a state of enhanced pain sensitivity. In addition, recent advancements in dynamic experimental paradigms have shown that CLBP may also be associated with systemic changes in pain modulation. Specifically, individuals with CLBP have demonstrated both enhanced pain facilitation [18] as well as decrements in pain inhibition [19] when compared to pain-free controls. Independent of chronic pain, experimental pain tests have also been used to determine the extent to which physical activity is associated with pain processing. A recent study of pain-free individuals found lower physical activity to be associated with heightened pain sensitivity [20,21]. Another study found the intensity of physical activity (e.g. light versus vigorous) played a role, as non-athletes demonstrated a lower pain tolerance compared to athletes when using standardized QST protocol [22]. To our knowledge, determining associations between physical activity and pain processing in the presence of CLBP has not been assessed. This association would provide important insight for health care providers and patients.

Therefore, the purpose of this study was to examine the relationship between the intensity of physical activity and experimental measures of pain processing in persons with CLBP, and to compare that with a group of pain-free individuals. We hypothe- sized that individuals with lower physical activity levels would demonstrate heightened pain sensitivity and decrement in pain modulation, as has been observed previously [20-22]. This study aimed to investigate the differential association between physical activity and pain processing based on the presence of CLBP, which could then be used to model future investigation into the prognostic value of physical activity to prevent and/or treat chronic pain conditions.

\section{Methods}

\subsection{Study design and participants}

Individuals aged 18-79 years with CLBP or painfree were recruited from the University of Florida (UF) and surrounding community via printed advertisements. Individuals with CLBP were considered for the study if they had experienced pain for at least 3 months that was equal to or greater than $40 / 100$ on a numeric pain rating scale. Exclusion criteria for both groups were: 1) prior surgery for low back musculoskeletal pain; 2) current use of narcotics; 3) uncontrolled hypertension; 4) diabetic neuropathy; 5) circulatory disorders; 6) history of cardiac events; 7) cardiac pacemaker; 8) epilepsy; 9) symptoms of nerve root involvement such as motor weakness and sensory disturbance; 10) pregnancy; or 11) psychiatric-related hospital admission within the past year. Additional exclusion criteria for individuals with CLBP were active treatment for low back pain either currently or within the past 1 month, or low back pain consequent to traumatic injury. All participants were screened in person for cognitive level using the Mini Mental State Examination (MMSE), with those scoring over a 23 considered for enrollment. The UF Institutional Review Board approved this study and all subjects were required to sign an informed consent form to participate.

Following consent, participants completed questionnaires pertaining to demographics and pain. Additionally, participants provided information on their level of physical activity including type, intensity, frequency, and duration. Experimental pain measures of pain processing were then completed which involved evocation of mechanical (pressure) and thermal (heat) pain stimuli. 


\subsection{Measurements}

\subsubsection{Physical activity levels}

A written questionnaire was completed by participants to assess and define physical activity level. Questions included the number and average duration of workouts per week, based on a priori definition of moderate (i.e., brisk walking, bicycling 5-9 mph, golf, swimming, lifting weights, etc.), or vigorous (running, bicycling $10 \mathrm{mph}+$, swimming laps, circuit weight training, competitive sports, etc.) physical activity level. Questions were derived from the International Physical Activity Questionnaire, which is a reliable and valid measure of activity [23]. However, questions were updated in order to align with current general physical activity guidelines and level of physical activity intensity [24-26]. A physical activity index was then calculated for each mode of intensity (i.e. moderate, vigorous) by multiplying the total number of workouts in the past week by minutes per workout.

\subsubsection{Pain processing measures}

Experimental pain was elicited to provide proxy measures of pain processing. The first was pain sensitivity, which can either be the individual's perception of pain threshold (when pain is first experienced) or tolerance (when pain is no longer tolerable). Only pain threshold was included in this study, which involved application of a pressure to the anterior lower leg by a force gauge (Wagner Instruments, Greenwich, CT). Force was applied at a rate of $1 \mathrm{Kg} / \mathrm{cm}^{2} /$ second until the individual verbally reported the onset of pain. Three measurements were collected and the average of all three measures provided the pain sensitivity measure for each individual.

The second pain processing measure assessed was pain modulation, or the change in pain intensity over time. Five heat pulses of the same temperature $\left(48^{\circ} \mathrm{C}\right)$ were applied to the bottom of the right foot for 0.5 seconds each (2.5 second interpulse interval) using a PATHWAY Model Advanced Thermal Stimulator (ATS) (Medoc Advanced Medical Systems, Ramat Yishai, Israel). Participants rated intensity of the second pain experienced after initial heat for each pulse using the numeric pain rating scale (NPRS; 0 equal to 'no pain', 100 equal to 'worst pain imaginable'), which occurs in between the heat pulses. The NPRS is a validated measure of how an individual perceives pain intensity [24]. Pain rating at the last heat pulse was then subtracted from pain rating at the first pulse, which provided the pain modulation score. This spe- cific test is referred to as temporal summation of second pain and has been used previously by our lab and others [27-29]. Moreover, this study is based on our understanding of pain modulation occurring at the spinal cord [30].

\subsection{Statistical analysis}

Analyses were completed using $\operatorname{IBM}^{\circledR} \operatorname{SPSS}^{\circledR}$ Statistics software, Version 21 (2012, IBM ${ }^{\circledR}$ Corp; Armonk, NY). To examine differences in intake characteristics, physical activity level, and experimental pain measures by condition (pain-free vs. individuals with CLBP), non-parametric Mann-Whitney U Test and chi-square tests were used. This was due to unequal sample sizes and heterogeneity of variance across groups.

Next, we assessed the association between moderate or vigorous physical activity level and pain sensitivity and modulation using univariate regression modeling. Separate models were created to assess the differential association based on condition (pain-free vs. individuals with CLBP). Alpha level was set at $p=0.05$ for all analyses.

\section{Results}

A total of 70 subjects participated in the study. Differences in demographics, physical activity, and pain processing measures are outlined in Table 1. Individuals with CLBP and pain-free groups were of similar age and gender $(p>0.05)$. Pain-free individuals had higher moderate $(U=688, p<0.05)$ and vigorous $(U=649, p<0.05)$ physical activity levels compared to individuals with CLBP. Moreover, pain-free individuals had lower pain sensitivity compared to individuals with CLBP, as evidenced by higher pressure pain threshold ( $p<0.05)$. Pain modulation levels were similar across groups $(p>0.05)$.

\subsection{Relationship between physical activity level and pain sensitivity}

Neither moderate nor vigorous physical activity were associated with pain sensitivity in pain-free $\left(\mathrm{R}^{2}=0.02, p>0.05\right)$ or individuals with $\operatorname{CLBP}\left(\mathrm{R}^{2}=\right.$ $0.01, p>0.05$ ) (Fig. 1). In addition, neither moderate nor vigorous physical activity was associated with pain modulation in individuals with $\operatorname{CLBP}\left(\mathrm{R}^{2}=0.01\right.$, $p>0.05)$. However, both moderate $\left(\mathrm{R}^{2}=0.49, p<\right.$ 
Table 1

Intake characteristics and differences in activity level and pain processing by group

\begin{tabular}{lccc}
\hline Group & $\begin{array}{c}\text { LBP }(n=49) \\
\text { Mean (SD) }\end{array}$ & $\begin{array}{c}\text { Pain-free }(n=21) \\
\text { Mean (SD) }\end{array}$ & P \\
\hline Age & $46.77(14.85)$ & $45.29(18.22)$ & 0.721 \\
Female (\%) & 69 & 57 & 0.421 \\
Moderate physical activity & & $3.14(2.56)$ & $\mathbf{0 . 0 3 7}$ \\
$\quad$ Number of Workouts (week) & $2.10(2.76)$ & $42.57(38.03)$ & $\mathbf{0 . 0 2 6}$ \\
$\quad$ Average Workout Duration (minutes) & $20.98(20.39)$ & $170.00(189.00)$ & $\mathbf{0 . 0 2 4}$ \\
$\quad$ Moderate Physical Activity Index** & $74.65(105.87)$ & $1.38(2.01)$ & 0.056 \\
$\quad$ Vigorous physical activity & $0.57(1.29)$ & $30.55(49.41)$ & $\mathbf{0 . 0 2 1}$ \\
$\quad$ Number of Workouts (week) & $8.47(16.81)$ & $93.38(166.60)$ & $\mathbf{0 . 0 4 0}$ \\
$\quad$ Average Workout Duration (minutes) & $21.94(66.77)$ & $7.49(2.86)$ & $\mathbf{0 . 0 1 7}$ \\
Vigorous Physical Activity Index** & & $0.48(16.23)$ & 0.354 \\
$\quad$ Pressure Pain Threshold (Kg/cm ${ }^{2} /$ second) & $5.65(2.86)$ & $4.06(14.05)$ & \\
$\quad$ Temporal Summation*
\end{tabular}

*Temporal summation (pain rating at $5^{\text {th }}$ pulse minus pain rating at $1^{\text {st }}$ pulse); ${ }^{* *}$ Moderate and Vigorous Physical Activity Index were calculated by multiplying the total number of workouts in the past week by minutes per workout.

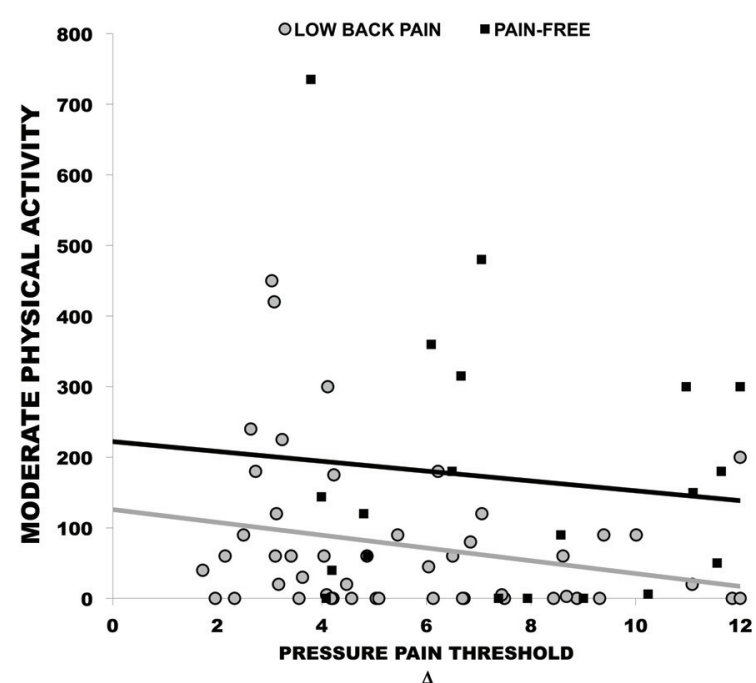

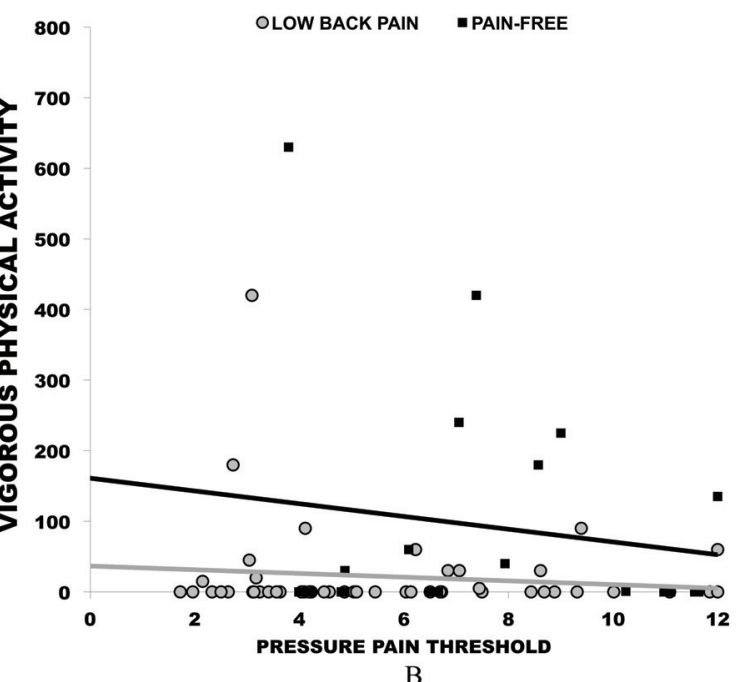

B

Fig. 1. Moderate and vigorous physical activity level and pain sensitivity relationship, by group.

$0.05)$ and vigorous $\left(\mathrm{R}^{2}=0.68, p<0.01\right)$ physical activity were negatively associated with pain modulation in pain-free individuals, meaning that higher physical activity was associated with higher pain modulation (Fig. 2).

\subsection{Extreme physical activity level data point}

Upon visual examination of the data, it was determined that an extreme score existed for moderate and vigorous physical activity levels amongst pain-free individuals (Figs 1 and 2). These data points were included in analyses for a number of reasons. First, no $a$ priori criteria were set to exclude extremes in activity level. Second, while the physical activity values of the pain-free individual in question are considered extreme values compared to other participants in this sample, the values are realistic for higher-level performance in other samples. Therefore, including these values provided a more representative sample of physical activity in the population.

\subsection{Post-Hoc analysis}

Since individuals across the entire lifespan were included in this study, a post-hoc analysis was performed to confirm physical activity differences by group, independent of age. As such, ages were included as a covariate using 1000-sample bootstrapped analysis of covariance modeling. After accounting for age, both 

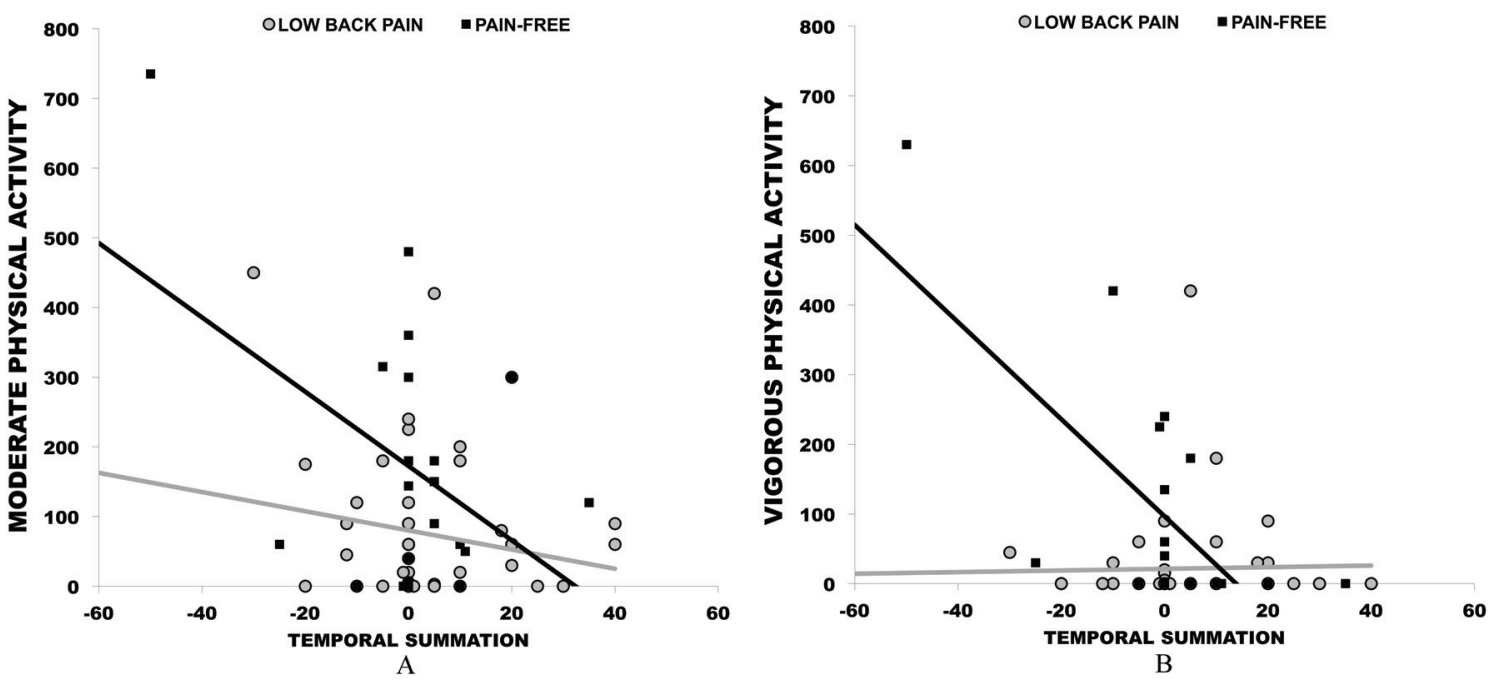

Fig. 2. Moderate and vigorous physical activity level and pain modulation relationship, by group.

moderate $\left(\mathrm{F}_{2,70}=3.80, p<0.05\right)$ and vigorous $\left(\mathrm{F}_{2,70}=3.80, p<0.05\right)$ physical activity levels remained higher in pain-free individuals compared to individuals with CLBP. Similarly, the influence of age on associations between physical activity and pain modulation were examined. However, univariate regression models found a lack of association between age and pain modulation for both pain-free individuals $\left(\mathrm{R}^{2}=\right.$ $0.02, p>0.05)$ and those individuals with CLBP $\left(\mathrm{R}^{2}=0.001, p>0.05\right)$. Therefore, we were confident age did not affect the observed associations.

\section{Discussion}

To our knowledge, this study is the first to examine associations between physical activity and experimentally-induced measures of pain processing- pain sensitivity and modulation- in both a pain-free and clinical pain cohort. Findings suggest that lower physical activity levels are associated with maladaptive pain modulation amongst pain-free individuals. In other words, those with lower physical activity were more sensitive to longer durations of experimentally-induced pain. The same associations were not found amongst individuals with CLBP. However, this study provides a novel preliminary indication of associations between physical activity levels and pain processing using laboratory correlates, which can be used to model future prognostic studies.

The key finding of this study was a lack of association between physical activity and pain processing amongst individuals with CLBP. These findings suggest that current levels of physical activity, once CLBP is present, do not appear to be related to current pain processing, or the influence of physical activity on pain lessens with pain chronicity. Alternatively, pre-established factors (e.g. psychosocial [31]) may have greater influence on CLBP than physical activity. Regardless, our findings do not suppress the potential preventative and/or interventional value of physical activity in the presence of CLBP, which should be investigated in future trials. Since this was a crosssectional study, we were only privy to individuals' physical activity level at one time point. It's possible that a stronger association exists between prolonged physical inactivity and pain sensitivity or modulation; which, in turn, contribute to the chronicity of pain. Further, it's possible that associations lie with lower levels of function (i.e. inability to perform activities of daily living) rather than higher levels of physical activity. It's worth noting that higher physical activity was not associated with decrements in pain processing, which is valuable since it suggests the potential for individuals with CLBP to engage in physical activity without adversely influencing central pain mechanisms. This too, requires further study for confirmation.

Independent of the lack of associations observed amongst the individuals in the CLBP cohort, the current research is promising as it aligns with previous work showing inverse relationships between physical activity and pain $[32,33]$. A recent paper by Naugle and Riley [33] examined the relationship between physical activity and pain sensitivity, much like the current study. Results were similar in that pain-free adults who engaged in more physical activity had improved de- 
scending pain modulation [33]. These findings may be representative of an association between physical activity level and pain modulation in that physical activity level directly corresponds to pain response. Mechanistically, physical activity levels may play a role in the neuroplasticity of the brain and its ability to mitigate pain input signals [34]. The concept of increased physical activity level and its inhibitory effects on pain modulation is similar to the bodies' natural negative feedback loop mechanism responsible for regulating and stabilizing several other body systems. For example, the ability of the body to maintain homeostasis (via temperature regulation) may be similar to regulatory effect of physical activity on pain processing. In other words, as one factor increases or decreases to a certain level, the other responds in the opposite direction. Therefore, if physical activity influences pain processing, exercise or interventions to promote physical activity may potentially prevent onset of chronic pain; or assist in chronic pain treatment.

There are multiple strengths to this study. First, we translated previous research examining physical activity and pain processing in pain-free individuals to those with an ongoing musculoskeletal pain condition [32]. Second, we used both a sensitivity measure (pressure pain threshold) and modulation measure (temporal summation of second pain), while prior research has predominantly used pain sensitivity measures [22,35]. It was important to include a pain sensitivity measure to link our findings to previous research. However, modulatory measures are considered a better indicator of pain processing [36]. To our knowledge, only two other studies have examined physical activity level and pain processing using a modulatory measure [32,33]; both of which found a negative relationship amongst pain-free individuals as we did here. An additional study strength was that our cohort was more representative of the variable activity levels seen across the general population, with some engaging in minimal physical activity and others at more extreme levels.

There are also limitations to this study. First, we used a cross-sectional analysis and therefore cannot ascertain the extent to which physical activity prevents CLBP, which should be the impetus for future research. Second, we did not include psychosocial factors, which prevented the comparative association to physical activity in the presence of experimentally-induced pain. Psychosocial factors, such as pain catastrophizing or pain-related fear, should be included in future prognostic trials as they have been found to influence pain chronicity [31]. Finally, while our sample included a heterogeneous cohort of physical activity (i.e. more representative of the general population), we may have been underpowered to examine differential association based on subgroups of population, such as those exclusively performing vigorous activity (e.g. triathletes). Physical activity subgroup analysis should be considered for future studies.

\section{Conclusions}

Our findings suggest that higher physical activity levels are associated with improved pain modulation among pain-free individuals. While multiple risk factors influence an individual's perception of pain, physical activity may be valuable from a rehabilitation perspective; as it is modifiable. Future research involving experimental pain measures will determine the extent to which physical activity prevents CLBP chronicity, as well as the ideal physical activity dosage for treatment once CLBP is present. Improved understanding of physical activity and CLBP will assist healthcare providers with optimizing prevention and treatment of CLBP.

\section{Acknowledgement}

We would like to thank Russell M. Bauer, PhD., ABPP, for his assistance with manuscript revision.

\section{Conflict of interest}

None to report.

\section{Funding sources}

This study was supported by funding from the University of Florida University Scholars Program and Department of Physical Therapy.

\section{References}

[1] Hoy D, March L, Brooks P, et al. The global burden of low back pain: Estimates from the Global Burden of Disease 2010 study. Ann Rheum Dis. 2014 Jun; 73(6): 968-74.

[2] Buchbinder R, Blyth FM, March LM, Brooks P, Woolf AD, Hoy DG. Placing the global burden of low back pain in context. Best Pract Res Clin Rheumatol. 2013; 27(5): 575-589. 
[3] Lautenbacher S, Kunz M, Strate P, Nielsen J, Arendt-Nielsen L. Age effects on pain thresholds, temporal summation and spatial summation of heat and pressure pain. Pain. 2005; 115(3): 410-418.

[4] Wiesenfeld-Hallin Z. Sex differences in pain perception. Gend Med. 2005; 2(3): 137-145.

[5] Pauli P, Rau H, Zhuang P, Brody S, Birbaumer N. Effects of smoking on thermal pain threshold in deprived and minimally-deprived habitual smokers. Psychopharmacology (Berl). 1993; 111(4): 472-476.

[6] Vassend O, Røysamb E, Nielsen CS. Five-factor personality traits and pain sensitivity: A twin study. Pain. 2013; 154(5): 722-728.

[7] Chen SM, Liu MF, Cook J, Bass S, Lo SK. Sedentary lifestyle as a risk factor for low back pain: A systematic review. Int Arch Occup Environ Health. 2009 Jul; 82(7): 797-806.

[8] Heneweer H, Staes F, Aufdemkampe G, van Rijn M, Vanhees L. Physical activity and low back pain: A systematic review of recent literature. Eur Spine J. 2011 Jun; 20(6): 826-45. doi: 10.1007/s00586-010-1680-7. Epub 2011 Jan 9. Review.

[9] Heneweer H, Vanhees L, Picavet HS. Physical activity and low back pain: a U-shaped relation? Pain. 2009 May; 143(12): 21-5.

[10] Strawbridge WJ, Deleger S, Roberts RE, Kaplan GA. Physical activity reduces the risk of subsequent depression for older adults. Am J Epidemiol. 2002 Aug 15; 156(4): 328-34.

[11] Nijs J, Van Houdenhove B, Oostendorp RA. Recognition of central sensitization in patients with musculoskeletal pain: Application of pain neurophysiology in manual therapy practice. Man Ther. 2010; 15(2): 135-141.

[12] Price DD, Staud R, Robinson ME, Mauderli AP, Cannon R, Vierck CJ. Enhanced temporal summation of second pain and its central modulation in fibromyalgia patients. Pain. 2002; 99(1-2): 49-59.

[13] Nir RR, Yarnitsky D. Conditioned pain modulation. Curr Opin Support Palliat Care. 2015 Jun; 9(2): 131-7.

[14] Clauw DJ, Williams D, Lauerman W, Dahlman M, Aslami A, Nachemson AL, Kobrine AI, Wiesel SW. Pain sensitivity as a correlate of clinical status in individuals with chronic low back pain. Spine (Phila Pa 1976).

[15] Giesecke T, Gracely RH, Grant MA, Nachemson A, Petzke F, Williams DA, Clauw DJ. Evidence of augmented central pain processing in idiopathic chronic low back pain. Arthritis Rheum. 2004 Feb; 50(2): 613-23.

[16] Puta C, Schulz B, Schoeler S, Magerl W, Gabriel B, Gabriel HH, Miltner WH, Weiss T. Enhanced sensitivity to punctate painful stimuli in female patients with chronic low back pain. BMC Neurol. 2012 Sep 21; 12: 98.

[17] Imamura M, Chen J, Matsubayashi SR, Targino RA, Alfieri FM, Bueno DK, Hsing WT. Changes in pressure pain threshold in patients with chronic nonspecific low back pain. Spine (Phila Pa 1976). 2013 Nov 15; 38(24): 2098-107.

[18] Tesarz J, Wolfgang E, Treede RD, Gerhardt A. Altered pressure pain thresholds and increased wind-up in adult chronic back pain patients with a history of childhood maltreatment: A quantitative sensory testing study. Pain. 2016 Apr 13. [Epub ahead of print]

[19] Mlekusch S, Neziri AY, Limacher A, Jüni P, Arendt-Nielsen L, Curatolo M. Conditioned Pain Modulation in Patients With Acute and Chronic Low Back Pain. Clin J Pain. 2016 Feb; 32(2): 116-21.

[20] Geva N, Defrin R. Enhanced pain modulation among triathletes: A possible explanation for their exceptional capabilities. Pain. 2013; 154(11): 2317-2323.
[21] Naugle KM, Riley 3rd, Joseph L. Self-reported Physica Activity Predicts Pain Inhibitory and Facilitatory Function. Medicine \& Science in Sports \& Exercise. 2014; 2013; 46: 622-629.

[22] Tesarz J, Schuster AK, Hartmann M, Gerhardt A, Eich W. Pain perception in athletes compared to normally active controls: A systematic review with meta-analysis. Pain. 2012; 153(6): 1253-1262.

[23] Craig CL, Marshall AL, Sjöström M, Bauman AE, Booth ML, Ainsworth BE, Pratt M, Ekelund U, Yngve A, Sallis JF, Oja P. International physical activity questionnaire: 12-country reliability and validity. Med Sci Sports Exerc. 2003 Aug; 35(8): 1381-95.

[24] Ferreira-Valente MA, Pais-Ribeiro JL, Jensen MP. Validity of four pain intensity rating scales. Pain. 2011 Oct; 152(10): 2399-404.

[25] Cdc.gov. Measuring Physical Activity Intensity [Internet] 2015 [cited 16 July 2015]. Available from: http://www.cdc. gov/physicalactivity/basics/measuring/index.html.

[26] Haskell WL, Lee IM, Pate RR, Powell KE, Blair SN, Franklin BA, Macera CA, Heath GW, Thompson PD, Bauman A. American College of Sports Medicine; American Heart Association. Physical activity and public health: updated recommendation for adults from the American College of Sports Medicine and the American Heart Association. Circulation. 2007 Aug 28; 116(9): 1081-93.

[27] Robinson ME, Bialosky JE, Bishop MD, Price DD, George SZ. Supra-threshold scaling, temporal summation, and aftersensation: relationships to each other and anxiety/fear. J Pain Res. 2010; 3: 25-32.

[28] Valencia C, Fillingim RB, George SZ. Suprathreshold heat pain response is associated with clinical pain intensity for patients with shoulder pain. J Pain. 2011; 12(1): 133-140.

[29] Valencia C, Kindler LL, Fillingim RB, George SZ. Investigation of central pain processing in shoulder pain: converging results from 2 musculoskeletal pain models. J Pain. 2012; 13(1): 81-89.

[30] Price DD, Hu JW, Dubner R, Gracely RH. Peripheral suppression of first pain and central summation of second pain evoked by noxious heat pulses. Pain. 1977; 3(1): 57-68.

[31] Crombez G, Eccleston C, Van Damme S, Vlaeyen JW, Karoly P. Fear-avoidance model of chronic pain: the next generation. Clin J Pain. 2012 Jul; 28(6): 475-83.

[32] Geva N, Defrin R. Enhanced pain modulation among triathletes: A possible explanation for their exceptional capabilities. Pain. 2013; 154(11): 2317-2323.

[33] Naugle KM, Riley 3rd, Joseph L. Self-reported Physical Activity Predicts Pain Inhibitory and Facilitatory Function. Medicine \& Science in Sports \& Exercise. 2014; 2013; 46: 622-629.

[34] Cotman CW, Berchtold NC. Exercise: A behavioral intervention to enhance brain health and plasticity. Trends Neurosci. 2002; 25: 295-301.

[35] Tesarz J, Schuster AK, Hartmann M, Gerhardt A, Eich W. Pain perception in athletes compared to normally active controls: A systematic review with meta-analysis. Pain. 2012; 153(6): 1253-1262.

[36] Arendt-Nielsen L, Yarnitsky D. Experimental and clinical applications of quantitative sensory testing applied to skin, muscles and viscera. J Pain. 2009; 10(6): 556-572. 